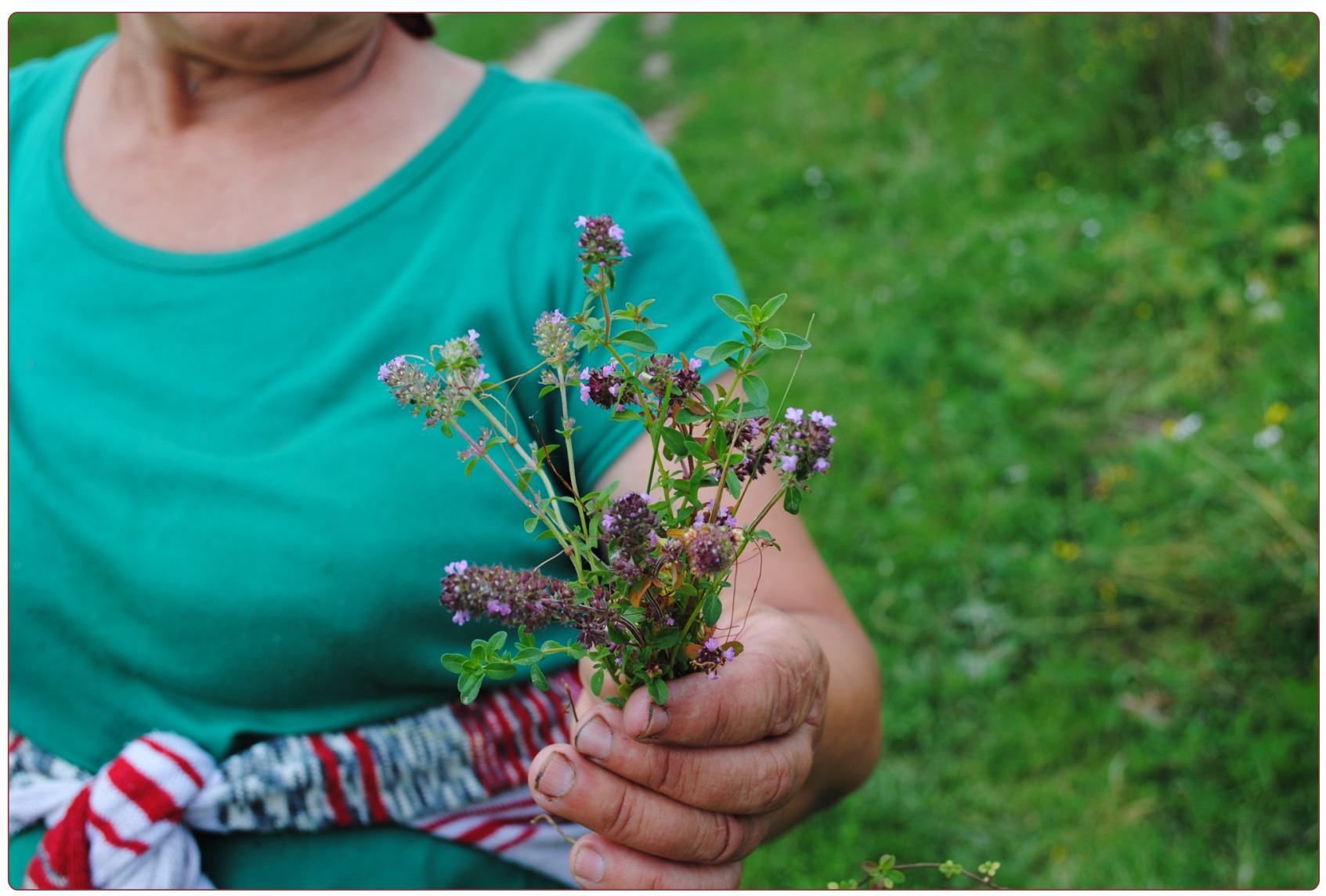

From economic survival to recreation: contemporary uses of wild food and medicine in rural Sweden, Ukraine and NW Russia

Stryamets et al.

Ciomed Central 


\title{
From economic survival to recreation: contemporary uses of wild food and medicine in rural Sweden, Ukraine and NW Russia
}

\author{
Nataliya Stryamets ${ }^{1,3^{*}}$, Marine Elbakidze ${ }^{1}$, Melissa Ceuterick ${ }^{2}$, Per Angelstam ${ }^{1}$ and Robert Axelsson ${ }^{1}$
}

\begin{abstract}
Background: There are many ethnobotanical studies on the use of wild plants and mushrooms for food and medicinal treatment in Europe. However, there is a lack of comparative ethnobotanical research on the role of non-wood forest products (NWFPs) as wild food and medicine in local livelihoods in countries with different socio-economic conditions. The aim of this study was to compare the present use of wild food and medicine in three places representing different stages of socio-economic development in Europe. Specifically we explore which plant and fungi species people use for food and medicine in three selected rural regions of Sweden, Ukraine and the Russian Federation.
\end{abstract}

Methods: We studied the current use of NWFPs for food and medicine in three rural areas that represent a gradient in economic development (as indicated by the World Bank), i.e., Småland high plain (south Sweden), Roztochya (western Ukraine), and Kortkeros (Komi Republic in North West Russia). All areas were characterised by (a) predominating rural residency, (b) high forest coverage, and (c) free access to NWFPs. A total of 205 in-depth semi-structured interviews were conducted with local residents in the three study areas. The collected NWFPs data included (1) the species that are used; (2) the amount harvested, (3) uses and practices (4) changes over time, (5) sources of knowledge regarding the use of NWFPs as wild food and medicine and (6) traditional recipes.

Results: In Sweden 11 species of wild plant and fungi species were used as food, and no plant species were used for medicinal purposes. In Ukraine the present use of NWFPs included 26 wild foods and 60 medicinal species, while in Russia 36 food and 44 medicinal species were reported.

Conclusions: In the economically less developed rural areas of Ukraine and Russia, the use of NWFPs continues to be an important part of livelihoods, both as a source of income and for domestic use as food and medicine. In Sweden the collection of wild food has become mainly a recreational activity and the use of medicinal plants is no longer prevalent among our respondents. This leads us to suggest that the consumption of wild food and medicine is influenced by the socio-economic situation in a country.

\section{Background}

Wild food and medicines are plant and fungal resources that grow in natural conditions and which are harvested or collected for the purpose of human consumption and used as food, dietary supplements and medical treatments $[1,2]$. For the purpose of this study animal

\footnotetext{
* Correspondence: Natalie.Stryamets@slu.se

'Swedish University of Agricultural Sciences, Forest-Landscape-Society Research Group, Faculty of Forest Sciences, School for Forest Management, PO Box 43, Skinnskatteberg SE 73921, Sweden

${ }^{3}$ Nature reserve "Roztochya", Sitchovuh Strilciv 7, Ivano-Frankove 81070, Ukraine

Full list of author information is available at the end of the article
}

resources were not included. 'Wild' refers to the fact that these species grow without being cultivated [3], however, semi-domesticated species are also included in this definition. The Food and Agriculture Organisation of the United Nations (FAO) proposed the term Non-Wood Forest Products (NWFPs) for goods of biological origin other than wood, derived from forests and other woodlands [4].

NWFPs have contributed to sustaining the livelihoods of people living in forest and woodland landscapes for centuries [5-8]. In Europe, the practice of collecting NWFPs for wild food and medicine has been declining 
due to economic development leading to urbanization, mass production of food and modern synthetically produced medicines $[3,9,10]$. However, as the health industry has developed over the last few decades, alarms about unhealthy additives in mass produced food have resulted in a renewed interest in wild food and medicine $[11,12]$. Wild food is considered pure, naturally healthy and rich in vitamins and antioxidants [13]. Moreover, wild plants and mushrooms play an important role as spices and accompaniments in traditional cuisines in Europe [3, 13-16]. While wild food is becoming a fashionable product $[3,17]$ in many European countries with a market economy, it is still an important resource for local livelihoods in countries in transition from a planned economy towards a market economy $[15,18-21]$. There is also a growing interest in traditional medicine in different parts of Europe [3, 13, 17, 22-25], even where collecting plants for medicinal purposes is no longer a widespread practice $[3,9,26,27]$.

There are many ethnobotanical studies on the use of wild plants and mushrooms for food and medicinal purposes in Europe [6, 15, 16, 19, 24, 28-43]. However, there is a lack of comparative ethnobotanical research on the role of NWFPs as wild food and medicine in local livelihoods in countries with different socio-economic conditions [13, 33, 44, 45].

The aim of this study is to document and analyse current uses of NWFPs as wild food and medicine in three European countries that are in different stages of socio-economic development. We used three rural case studies, located in Sweden, Ukraine and the Russian Federation that represent a gradient in economic development from market economy with intensive industrial management of forests and agricultural land to countries in transition from planned economy to market economy with still existing traditional use of forest and agricultural landscapes [46, 47]. The following research questions will be answered here: Which plant and fungi species do rural people use for food and medicine in the selected rural regions of Sweden, Ukraine and Russia? Do people keep traditional knowledge related to wild wood and medicine? Are there any similarities or differences in the use of wild food and medicine among places with different socio-economic conditions?

\section{Methods}

\section{Study areas}

The Småland high plain (hereafter Småland) in south Sweden, the Roztochya upland region (hereafter Roztochya) in western Ukraine and the Kortkeros rayon (municipality) (hereafter Kortkeros) in the Komi Republic in North West (NW) Russia were selected as case study areas. The selected study areas are all dominated by rural residency, have a high percentage of forest coverage, and free access to forest that provide NWFPs. In all three study areas NWFPs have traditionally been important sources of wild food and medicine for centuries $[6,15,16$, $19,35,48-50]$.

Småland $\left(56^{\circ} 52^{\prime}-57^{\circ} 26^{\prime} \mathrm{N}\right.$ and $\left.14^{\circ} 43^{\prime}-15^{\circ} 04^{\prime} \quad \mathrm{E}\right)$ (Fig. 1) encompasses $1792 \mathrm{~km}^{2}$. Boreal forest forms the main land cover, and occupies more than $50 \%$ of the study area. Forests are dominated by Scots pine [Pinus sylvestris L.] and Norway spruce [Picea abies (L.) Karst.]. The study area is surrounded by hemiboreal forests and temperate lowland deciduous forests with beech [Fagus silvatica L.] in the south. In the past this forestdominated landscapes was used for traditional animal husbandry and farming [51, 52]. Non-industrial private owners own $80-85 \%$ of the forests in the study area [53]. Other main forest owners are the state-owned forest company Sveaskog Co., municipalities and the Swedish Church. This study area includes 22 parishes with an average population density of 53 inhabitants per $\mathrm{km}^{2}$, but with only 13 per $\mathrm{km}^{2}$ in rural areas [54]. The population trend is negative, especially in rural areas that presently host $26 \%$ of the population in the study area [54]. Nevertheless, unemployment rates are lower than the Swedish average. As everywhere in Sweden, the social security and health care systems are well developed with insurance systems, unemployment funds and support grants available for all people in need [55].

Roztochya $\left(50^{\circ} 06^{\prime} \mathrm{N}-49^{\circ} 06^{\prime} \mathrm{N}\right.$ and $23^{\circ} 20^{\prime} \mathrm{E}-23^{\circ} 54^{\prime}$ E) is situated in the temperate lowland forest ecoregion, and covers $992 \mathrm{~km}^{2}$ (Fig. 1). Forests cover about $44 \%$ of the study area. Agricultural land, cultural woodlands and settlements make up the rest. The forest types are very diverse and range from dry sites with Scots pine to mesic sites with beech to wet sites with ash [Fraxinus excelsior L.] and black alder [Alnus glutinosa L.] [56]. Recently, a major part of this territory was designated as a MAB UNESCO Biosphere Reserve "Roztochya" [57, 58]. There are 120 settlements in Roztochya with about 60,000 inhabitants (Yavorivskiy and Zhovkivskiy rayons). The population density is about 80 persons per $\mathrm{km}^{2}$ [59]. The level of official unemployment for 2013 was $1.6-2 \%$, (yet actual rates might be much higher) which is a major problem in the region [60]. The healthcare system and its health centres are funded by the state and are theoretically free to all citizens. However, the cost of medicines and treatments often has to be covered by the patient. There are also some private health care centres in the area. However, their services are exceedingly expensive for many people in rural areas.

Kortkeros $\left(60^{\circ} 45^{\prime} \mathrm{N}-62^{\circ} 50^{\prime} \mathrm{N}\right.$ and $\left.50^{\circ} 45^{\prime} \mathrm{E}-53^{\circ} 30^{\prime} \mathrm{E}\right)$ occupies $19,700 \mathrm{~km}^{2}$ (Fig. 1). The forest cover is approximately $90 \%$ [61]. The boreal forest is formed by Scots pine and Norway spruce in later successional stages, but with a domination of birch [Betula spp.] and aspen 


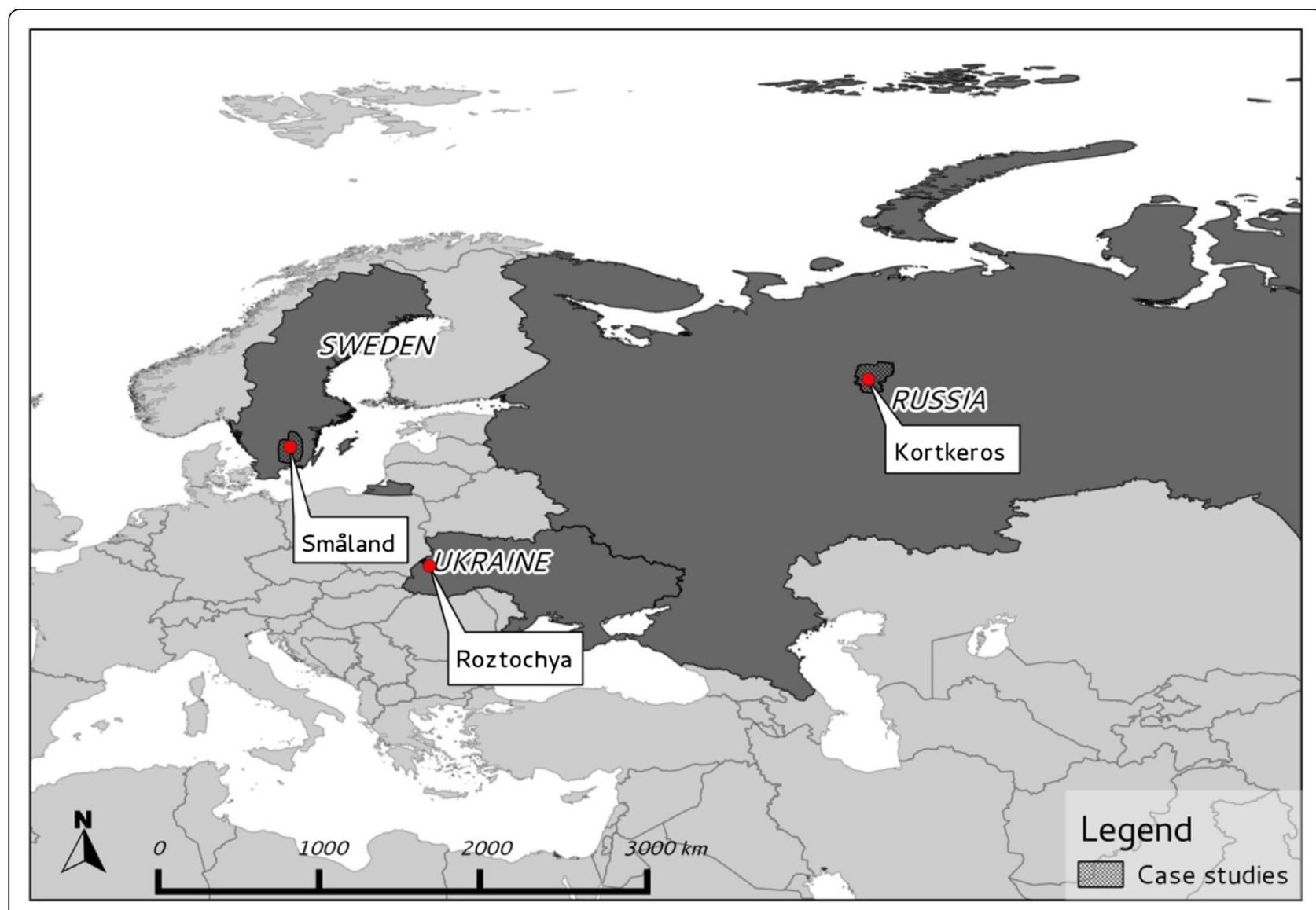

Fig. 1 Case studies in Sweden, Ukraine and NW Russia

[Populus tremula L.] in young and middle-aged forests. The human population density is low, less than one person per $\mathrm{km}^{2}$, and most of the population lives in rural areas. There are 60 villages with a total of 19,200 inhabitants [62]. The low population density in the area is both due to a low birth rate and a high emigration rate. A high level of unemployment ( $21 \%$ officially) is a main problem [62]. Health care is free to all citizens but accessibility is low. The only regional hospital is located in the village of Kortkeros. Patients have to cover all costs for medicines themselves and the prices are relatively high.

\section{Interviews}

In total 205 semi-structured interviews [63, 64] were conducted with local people in the three study areas (60 in Småland, 90 in Roztochya and 55 in Kortkeros) during a total of 6 months of field work from 2010 to 2013. Local inhabitants were selected for interviews using a convenience sample in the three study areas. Interviewees were chosen among people who were met in the forest near villages or on the village's street. First, the study was introduced and oral consent was obtained, along with a permit to record the interview. National or ethnic belonging was not asked, but was recorded if the respondent provided that information voluntarily. The uneven number of respondents among the study areas was due to the fact that the diversity of answers among the respondents in Roztochya was higher in comparison with the Swedish and the Russian study areas, and a larger sample of respondents was required in order to reach the data saturation point when no new information was provided by additional interviews [65].

The semi-structured interview manual consisted of open-ended questions and specific questions for confirmation. Questions focused on (1) the species of plants and fungi that are harvested from the forest; (2) the amount harvested, (3) current personal uses and practices, and (4) changes of over time, (5) sources of knowledge regarding the use of those species as wild food and medicine, (6) traditional recipes of dishes and medicine from NWFPs. Using open-ended questions, interviewees had full freedom to talk about NWFPs from their own perspective. Respondents were asked to divide NWFPs that they used into wild food and medicine. This was asked because the line between wild food and medicine is not always easy to draw and NWFPs are often 
used both as medicine and food. Pieroni et al. [28] conceptualised a continuum, ranging from species that are only used for food, through multipurpose food and medicinal species, to exclusively medicinal species. In addition, species that are consumed without any unique specification of their assumed health benefit, because they are considered to be 'healthy' in general, are called 'functional foods' [30]. The project followed the ethical guidelines outlined by the American Anthropological Association [66] and the International Society of Ethnobiology [67].

All respondents were interviewed in their native language (Swedish, Ukrainian and Russian) by the first author. In Småland the interviews were conducted with the help of an interpreter. The interviews lasted between 15 and $110 \mathrm{~min}$ and were digitally recorded (Olympus DM-20), and then fully transcribed. The interview transcripts were analysed for emergent themes related to the research questions.

\section{Identifying plant species and uses}

Plant species were identified during interviews in the field by the first author who has been botanically trained at university level. Interviews took place either in the forest or at the homes of peoples. If interviews took place inside and the local plant/fungi name was unknown to the researcher, additional information or local synonyms were asked for during the interviews. In Roztochya and Småland, respondents went outside during interviews and pointed out the discussed species, if needed. This was possible due to the seasonal timing of the interviews. In Kortkeros, however, the fieldwork was done in late autumn, therefore additional questions had to be asked to clarify the names of species, some respondents used books with pictures and botanical names of the plants to identify the plants. Hence, since it was possible to identify all species through visual identification in the field or by asking additional questions, no voucher specimens were collected. Latin names of plant species were further verified based on Flora Europea [68] and International Plant Name Index [67]. Names of mushrooms species were verified based on SPECIES 2000 database [69]. An inventory of NWFPs harvested and used as wild food and medicine was compiled, based on all species that were mentioned during the interviews. We included wild species, semi-domesticated and domesticated wild species, such as Mentha spp. and Sambucus nigra L. in the study [3].

\section{Results}

\section{Wild food used at home Plant species}

Respondents in all three case studies used a wide range of plant species as food. Harvested NWFPs were used both fresh and processed. Wild fruits and mushrooms were considered to be good for diversifying everyday meals.

Generally, all respondents collected wild fruits, specifically berries. The amount of collected species differed between informants and study areas (Table 1). In both the Ukrainian and Russian study areas the amount of collected fruits per household was higher than in the Swedish study area. But in some cases in Småland, respondents collected large amounts of wild fruits for consumption during winter time, as well as to share with friends and relatives. In both Roztochya and Kortkeross the respondents stated that it was hard to collect fruits (including berries), and define quantities as the harvest was dependent on yearly yields. Fruits were consumed fresh (sometimes as juice) or prepared to preserve (as we will discuss further).

Besides fruits (berries), birch sap was another popular form of wild food in the Russian and the Ukrainian study areas. In both areas, respondents collected the sap of birch [Betula pendula Roth. and B. pubescens Ehrh.] (from 3 to $10 \mathrm{l}$ per household) for personal needs, as "a healthy and tasty drink".

In addition, in Roztochya during spring times young leaves from common nettle [Urtica dioica L.] and Primula spp. (both Primula veris and P. elatior) were also collected and used in salads and soups. Common elder flowers [Sambucus nigra L.] were used to make a sparkling drink. Both Rumex spp. and Urtica dioica were used in soups by respondents in Roztochya. Rumex acetosa are commonly cultivated and Urtica dioica grows in the gardens, therefore, respondents did not view those species as wild food.

\section{Fungi}

Fungi were popular wild food in all three areas. The majority of respondents in Småland and all respondents in Roztochya and Kortkeros collected mushrooms (see Tables 2, 3 and 4). In Småland the most popular harvested mushrooms were: chanterelle [Cantharellus cibarius Fr.] and funnel chanterelle [Craterellus tubaeformis (Fr.) Quél.]. In Roztochya the most popular mushrooms were Boletus spp. ${ }^{1}$ Respondents also liked to collect sheep's head [Both Polyporus umbellatus (Pers.) Fr. and Grifola frondosa (Dicks.) Gray], which is a Red Book listed species in Ukraine [70]. The most popular species in Kortkeros was Lactarius spp.

\section{Preparing for winter time: preserves}

In order to overcome the season-dependent supply of wild foods, people in all three areas made preserves for winter times, such as different jams, juices and marmalades from wild fruits or marinated mushrooms. However, the diversity and quantities of preserves were higher in the Russian and Ukrainian study areas than in 
Table 1 The number and amount of collected fruits and fungi species in the study areas

\begin{tabular}{llll}
\hline & Småland, Sweden & Roztochya, Ukraine & Kortkeros, Russian Federation \\
\hline Maximum number of collected berry species per person & 8 & 14 & 12 \\
Average number of collected berry species per person & 3 & 8 & 6 \\
Maximum number of collected fungi species per person & 9 & 13 & 12 \\
Average number of collected fungi species per person & 3 & 6 & 6 \\
Amount of collected fruits (average/max), litres family/year: & & $10 / 35$ & $20 / 65$ \\
Vaccinium myrtillus L. & $2-5 / 50$ & $2 / 5$ & 20 \\
Vaccinium vitis-idaea L. & $2-5 / 50$ & $10 / 15$ & - \\
Rubus spp. & $2 / 5$ & $10 / 20$ & 1 \\
Rubus idaeus L. & - & $1-2 / 50$ & 30 \\
Fragaria vesca L. & - & $1 / 2$ & 10 \\
Vaccinium oxycoccos L. & - & $3 / 130$ & $3 / 200$ \\
Rubus chamaemorus L. & $1 / 50$ & $3 / 130$ & $3 / 30$ \\
Amount of collected mushrooms (average/max), kg family/year: & $1 / 6$ & $1 / 5$ & $5 / 45$ \\
Boletus edulis Bull. & $1 / 50$ & - & $15 / 200$ \\
Cantharellus cibarius Fr. & - & & 20 \\
Lactarius resimus Fr. & & & 12 \\
\hline
\end{tabular}

the Swedish case study. In Småland, some respondents made preserves from mushrooms, bilberries and cowberries for personal consumption during winter times. Chanterelles were frozen and consumed during winter time and funnel chanterelle were used dried for stews and sauces in the Swedish study area. However, most respondents collected chanterelle and funnel chanterelle once or twice per year for immediate cooking. Cowberry jam was used as an accompaniment to meat dishes and pancakes in Småland.

Table 2 Use of mushrooms in Roztochya (Ukraine)

\begin{tabular}{|c|c|c|c|c|}
\hline Family & Species & English name & Local names & Food use \\
\hline Agaricaceae & Macrolepiota spp. & Parasol mushroom & $\begin{array}{l}\text { Гриб-парасолька, парасолька, } \\
\text { гриб-зонтик }\end{array}$ & Whole mushroom \\
\hline Agaricaceae & Agaricus campestris L. & Field mushroom & Печериця, шампіньйон & Whole mushroom \\
\hline Boletaceae & Boletus edulis Bull. & Penny bun & $\begin{array}{l}\text { Білий гриб, боровик, білий, } \\
\text { справжній гриб }\end{array}$ & Whole mushroom \\
\hline Boletaceae & $\begin{array}{l}\text { Leccinum aurantiacum } \\
\text { (Bull.) Gray }\end{array}$ & Red-capped scaber stalk & $\begin{array}{l}\text { Підосиковик, червоноголовець, } \\
\text { червнонюх, підосичник }\end{array}$ & Whole mushroom \\
\hline Boletaceae & $\begin{array}{l}\text { Leccinum scabrum (Bull.) } \\
\text { Gray }\end{array}$ & Scaber stalk & $\begin{array}{l}\text { Підберезовик, козар, козарик, } \\
\text { бабка }\end{array}$ & Whole mushroom \\
\hline Boletaceae & Boletus badius (Fr.) Fr. & Bay bolete & Польський гриб & Whole mushroom \\
\hline Boletaceae & Boletus chrysenteron Bull. & Red cracking bolete & Моховик, решітка & Whole mushroom \\
\hline Cantharellaceae & Cantharellus cibarius Fr. & Chanterelle & Лисичка & whole mushroom \\
\hline Meripilaceae & $\begin{array}{l}\text { Grifola frondosa (Dicks.) } \\
\text { Gray }\end{array}$ & Sheep's head & $\begin{array}{l}\text { Бараняча голова, баранячка, } \\
\text { бараньоха }\end{array}$ & Whole mushroom \\
\hline Polyporaceae & $\begin{array}{l}\text { Polyporus umbellatus } \\
\text { (Pers.) Fr. }\end{array}$ & Sheep's head & $\begin{array}{l}\text { Бараняча голова, баранячка, } \\
\text { бараньоха }\end{array}$ & Whole mushroom \\
\hline Morchellaceae & $\begin{array}{l}\text { Morchella esculenta (L.) } \\
\text { Pers. }\end{array}$ & Common morel & Зморшок, сморшок & Whole mushroom \\
\hline Physalacriaceae & $\begin{array}{l}\text { Armillaria mellea (Vahl) P. } \\
\text { Kumm. }\end{array}$ & Honey fungus & Опеньок & Whole mushroom \\
\hline Russulaceae & Lactarius resimus (Fr.) Fr. & & Груздь & Whole mushroom \\
\hline Russulaceae & Russula spp. & Russula & Сироїжки & Whole mushroom \\
\hline Suillaceae & Suillus luteus (L.) Roussel & Slippery jack & Маслюк & Whole mushroom \\
\hline
\end{tabular}


Table 3 Use of mushrooms in Kortkeros (Russian Federation)

\begin{tabular}{|c|c|c|c|c|}
\hline Family & Species & English name & Local names & Food use \\
\hline Agaricaceae & Macrolepiota spp. & Parasol mushroom & Гриб-зонтик, зонтик & Whole mushroom \\
\hline Boletaceae & Boletus edulis Bull. & Penny bun & Белый гриб, боровик & Whole mushroom \\
\hline Boletaceae & $\begin{array}{l}\text { Leccinum aurantiacum } \\
\text { (Bull.) Gray }\end{array}$ & Red-capped scaber stalk & $\begin{array}{l}\text { Подосиновик красный, } \\
\text { подосиновик, красноголовик }\end{array}$ & Whole mushroom \\
\hline Boletaceae & $\begin{array}{l}\text { Leccinum scabrum (Bull.) } \\
\text { Gray }\end{array}$ & Scaber stalk & $\begin{array}{l}\text { Подберёзовик обыкновенный, } \\
\text { подберёзовик, чёрный }\end{array}$ & Whole mushroom \\
\hline Boletaceae & Boletus chrysenteron Bull. & Red cracking bolete & Моховик & Whole mushroom \\
\hline Cantharellaceae & Cantharellus cibarius Fr. & Chanterelle & Лисичка обыкновенная, лисичка & Whole mushroom \\
\hline Discinaceae & $\begin{array}{l}\text { Gyromitra esculenta } \\
\text { (Pers.) Fr. }\end{array}$ & False morels & Строчок & Whole mushroom \\
\hline Morchellaceae & $\begin{array}{l}\text { Morchella esculenta (L.) } \\
\text { Pers. }\end{array}$ & Common morel & Сморчок & Whole mushroom \\
\hline Physalacriaceae & $\begin{array}{l}\text { Armillaria mellea (Vahl) } \\
\text { P. Kumm. }\end{array}$ & Honey fungus & Опёнок, собачий гриб & Whole mushroom \\
\hline Russulaceae & Lactarius pubescens Fr. & Downy milk cap & Волнушка белая, волнушка & Whole mushroom \\
\hline Russulaceae & Lactarius resimus (Fr.) Fr. & & Груздь настоящий, груздь & Whole mushroom \\
\hline Russulaceae & $\begin{array}{l}\text { Lactarius torminosus } \\
\text { (Schaeff.) Gray }\end{array}$ & Woolly milkcap & Волнушка розовая & Whole mushroom \\
\hline Russulaceae & Russula spp. & Russula & Сироежки & Whole mushroom \\
\hline Suillaceae & Suillus luteus (L.) Roussel & Slippery jack & $\begin{array}{l}\text { Маслёнок обыкновенный, } \\
\text { маслята, маслёнок }\end{array}$ & Whole mushroom \\
\hline Suillaceae & Suillus bovinus (L.) Roussel & Jersey cow mushroom & Козляк & Whole mushroom \\
\hline Tricholomataceae & $\begin{array}{l}\text { Tricholoma equestre (L.) } \\
\text { P. Kumm. }\end{array}$ & Yellow knight & Зеленушка & Whole mushroom \\
\hline
\end{tabular}

In both the Ukrainian and Russian cases jam, marmalade and juice made of bilberries, wild strawberries and raspberries were popular among respondents. In Roztochya preserves were additions for the everyday menu. Boletus species were dried or marinated (pickled) and dishes containing this species were considered a delicacy. Armillaria spp. was mainly used marinated. Russula spp. was used only fresh for immediate cooking.

Furthermore, respondents shared recipes for homemade honey made of dandelion flowers [Taraxacum officinale Weber ex Wiggers], which is considered to be a very tasty jam. The Ukrainian respondents mentioned that due to the increased availability of good quality freezers, they preferred deep-freezing mushrooms over marinating as a preservation technique for winter times.

All respondents in the Russian study area salted and pickled Lactarius spp., while marinated Boletus spp. was popular as well. Lacto-fermented Lactarius spp. were reported as a delicacy and a part of rural cuisine for centuries. During recent years, due to the availability of freezers, frozen mushrooms were also widely used. Respondents pointed out that Cantharellus cibarius was not used by native Komi people in the past, and local consumption started recently. Russian respondents froze cowberries and cranberries to preserve them for winter times. The frozen fruits would 'taste as if they were freshly picked'. The majority of respondents in the

Table 4 Use of mushrooms in Småland (Sweden)

\begin{tabular}{lllll}
\hline Family & Species & English name & Local names & Food use \\
\hline Agaricaceae & Macrolepiota spp. & Parasol mushroom & Fnasig fjällskivling & Whole mushroom \\
Boletaceae & Boletus edulis Bull. & Penny bun & Karljohan & Whole mushroom \\
Boletaceae & Leccinum aurantiacum & Red-capped scaber & Aspsopp & Whole mushroom \\
Boletaceae & (Bull.) Gray & stalk & Brun aspsopp & Whole mushroom \\
Cantharellaceae & Leccinum scabrum (Bull.) & Scaber stalk & & Whole mushroom \\
Cantharellaceae & Cantharellus cibarius Fr. & Chanterelle & Kantarell & Whole mushroom \\
\hline
\end{tabular}


Russian study area prepared jam from cloudberries (Rubus chamaemorus L.), which they considered a delicacy. Dried fruits of Prunus padus were used for a traditional drink, called 'lyazj'. Wild Ribes nigrum and R. rubrum were used for jam and marmalade. Traditional cookies called 'shanechky' or 'shangy' were made with jam made of bilberries or cowberries.

\section{Past versus present}

For Swedish respondents the use of NWFPs is not as important as it used to be for earlier generations. Interviewees stated that 20 years ago it was more common to pick different wild fruits and mushrooms for food. Respondents pointed out that, nowadays, one can buy everything from a store. Among Swedish respondents it is common to give collected fruits as a present to friends or family members. Contrarily, for Ukrainian and Russian respondents the use of NWFPs also played an important role for their livelihoods.

Additionally, respondents in the Ukrainian study area mentioned that it is still a tradition to cook dishes using NWFPs for religious holidays such as Christmas. For Christmas, respondents mentioned a traditional vegetarian dinner, including the special dish varenyky (or ravioli, i.e., small squares of pasta containing a mixture of mushrooms, potatoes or cheese) with mushrooms (called vushka) served with beetroot soup (borzch), and potatoes with mushrooms sauce. This tradition is considered important even by respondents who no longer collected mushrooms as a subsistence activity. In Roztochya one respondent named Chenopodium spp., which was used as "famine food" during the times of World War II.

The forest as apothecary: wild species used as medicine In Småland, local people no longer collected medicinal herbs for curative treatments. Respondents often mentioned that they did not have enough knowledge about medicinal plants and fungi. Instead they go to the forest for recreational activities to relief stress, and to get energized. Participants mentioned that they collected wild fruits because they were rich in vitamins. These functional food uses could be considered medicinal in a broad (preventive) sense. People in all study areas considered fruits to be rich in vitamins and thus good for one's health. Respondents in the Ukrainian and Russian study areas collected fruits for their kids because they considered it a natural and healthy product. Thus, some species were only used as food (Tables 5 and 6), while other species were used both as wild food and medicine. This example of functional foods illustrates that it is often hard to distinguish between the use of NWFPs as wild food or medicine. Some species were exclusively used as medicine (such as Arctium lappa L., Tussilago farfara L., Plantago major L., Chamomilla recutita (L.) Rauschert, Elymus repens (L.) Gould).

Unlike in Sweden, in the Ukrainian and Russian study areas, the collection of medicinal herbs was as popular as collecting wild food. The medicinal herbs used in Roztochya and Kortkeros are presented in Tables 5 and 6. The most popular species were raspberries [Rubus idaeus L.], guelder rose [Viburnum opulus L.], common hawthorn [Crataegus spp.] and rowan [Sorbus aucuparia L.], wild strawberries [Fragaria vesca L.], common nettle and dog rose [Rosa canina L.]. Different plant parts were used, such as the flowers of linden [Tilia cordata Mill.], the buds of birch tree [Betula pendula Roth.] and the leaves of common nettle.

In the Russian study area, the most used medicinal herbs were: cloudberries, cowberries, bilberries, raspberries, St John's wort [Hypericum perforatum L.] and greater plantain [Plantago major L.]. The Russian respondents considered it important to use medicinal herbs during winter times to prevent flu and common colds.

Respondents in both Ukraine and Russia used medicinal herbs either as infusions or they prepared different kinds of tinctures for 'promoting health'. Most medicinal plants were collected, dried in a dark place, and used for making an infusion. As a rule, dried herbs had to be infused for one hour before drinking. For preparing a tincture, additional ingredients were used, such as alcohol, sugar or honey. In addition, some medicinal treatments from animal origin were used in Kortkeross. Fat from the brown bear [Ursus arctos, Linnaeus, 1758] was used as a treatment for many different ailments. Fat from the European badger [Meles meles, Linnaeus, 1758] and marmot [Marmot spp.] were known to be used to treat tuberculosis.

In general, respondents in the Ukrainian and Russian study areas preferred to use medicinal herbs against certain illnesses rather than allopathic medicine from the pharmacy or shops. They also considered herbal remedies to be more environmentally friendly than pharmaceuticals. Moreover, interviewees often stated that their income was low and accordingly, pharmaceuticals were considered very expensive, while the cost associated with collecting medicinal herbs was much lower. Interestingly respondents reported their active use of medicinal herbs had increased since the collapse of the Soviet Union in 1991. Ukrainian respondents also mentioned cultural traditions and knowledge as important reasons for collecting wild food.

The most common ailments cured with herbal remedies in the Ukrainian case were flu, cough and gastrointestinal problems (Table 5). In addition, herbal remedies were used as vitamins, immuno-stimulants and cosmetics. Medicinal herbs were also used to treat chronic diseases 
Table 5 Use of medicinal herbs in the Roztochya (Ukraine)

\begin{tabular}{|c|c|c|c|c|c|c|c|c|}
\hline Family & Species & English name & Local names & Part used & Mode of preparation & Medicinal use & Food use & Level of use \\
\hline APOCYNACEAE & Vinca minor $\mathrm{L}$. & Lesser periwinkle & $\begin{array}{l}\text { Барвінок малий, } \\
\text { барвінок }\end{array}$ & $f l$, ap & Tincture & $\begin{array}{l}\text { Eye problems, blood cleaning, } \\
\text { blood coagulation }\end{array}$ & - & + \\
\hline ARACEAE & Acorus calamus $\mathrm{L}$. & Calamus & Aїр, шавар & r & Tincture & $\begin{array}{l}\text { Problems with gastrointestinal } \\
\text { tract, gangrene }\end{array}$ & - & + \\
\hline ARISTOLOCHIACEAE & Asarum europaeum L. & European wild ginger & Копитняк & $1, r$ & Tincture & $\begin{array}{l}\text { Heart, bronchiae, nervous } \\
\text { problems }\end{array}$ & - & + \\
\hline BETULACEAE & Betula spp. & Birch & Береза & $\mathrm{bd}, \mathrm{sa}$ & tea, tincture & $\begin{array}{l}\text { Kidneys, cosmetic for hair, skin, } \\
\text { vitamins }\end{array}$ & - & + \\
\hline BORAGINACEAE & Symphytym officinale L. & Comfrey & Живокіст & ap & $\begin{array}{l}\text { Liquids with alcohol, } \\
\text { tincture }\end{array}$ & Arthritis ('when hands hurt') & - & + \\
\hline CANNABACEAE & Humulus lupulus L. & Hops & Хміль & $\mathrm{fr}$ & Infusion, boiled tincture & $\begin{array}{l}\text { Cosmetic use (hair conditioner), } \\
\text { hair loss, blood pressure, liver } \\
\text { disease }\end{array}$ & - & + \\
\hline \multirow[t]{2}{*}{ CAPRIFOLIACEAE } & Sambucus nigra L. & Elderberry & Бузина чорна & $\mathrm{fl}$ & Tincture & Cough & - & + \\
\hline & Viburnum opulus L. & Guelder rose & Калина звичайна & $b$ & Tincture & $\begin{array}{l}\text { Blood pressure, vitamins, } \\
\text { immunity improving, cough }\end{array}$ & Fruits & + \\
\hline \multirow[t]{11}{*}{ COMPOSITAE } & Achillea millefollium L. & Yarrow & Деревій, тисячолистник & $\mathrm{fl}, \mathrm{ap}$ & Tincture & $\begin{array}{l}\text { Wound treatment, haemostatic, } \\
\text { blood coagulation }\end{array}$ & - & + \\
\hline & Anthemis maritima $\mathrm{L}$. & Maritime chamomile & Романець & $\mathrm{fl}$ & Infusion & Anthelmintic & - & + \\
\hline & Arctium lappa $\mathrm{L}$. & Greater burdock & Лопух & r & Infusion, tincture & $\begin{array}{l}\text { Cosmetics, for 'nice shining' } \\
\text { hair and hair growth }\end{array}$ & - & + \\
\hline & Artemisia absinthium L. & Wormwood & Полин, полин гіркий & ap & tincture & $\begin{array}{l}\text { Stomach ailments, anthelmintic, } \\
\text { fleas }\end{array}$ & - & + \\
\hline & Bidens tripartita $\mathrm{L}$. & Three-lobe beggarticks & Череда & । & Bath & Skin disease, for babies bath & - & + \\
\hline & Calendula officinalis L. & Marigold & Календула, нагідки & $\mathrm{fl}$ & Infusion, tincture & Antiseptic, tonsillitis & - & + \\
\hline & $\begin{array}{l}\text { Chamomilla recutita } \\
\text { (L.) Rauschert }\end{array}$ & Chamomile & Ромашка, рум'янок & $\mathrm{fl}, \mathrm{ap}$ & Infusion, tincture & $\begin{array}{l}\text { Stomach ailments,epatitis, } \\
\text { cosmetics } \\
\text { (skin softness), to treat burns }\end{array}$ & - & ++ \\
\hline & Centaurea cyanus L. & Cornflower & Волошки сині & $\mathrm{fl}$ & Infusion & Urinary tract & - & + \\
\hline & $\begin{array}{l}\text { Helichrysum arenarium } \\
\text { (L.) Moench }\end{array}$ & Dwarf everlast & Безсмертник, цмин & $\mathrm{fl}$ & Infusion & $\begin{array}{l}\text { Antiseptic, tuberculosis, } \\
\text { hepatitis }\end{array}$ & - & + \\
\hline & $\begin{array}{l}\text { Taraxacum officinale } \\
\text { Weber ex Wiggers }\end{array}$ & Dandelion & Кульбаба, & $\mathrm{fl}$ & $\begin{array}{l}\text { Liquids with alcohol, } \\
\text { tincture }\end{array}$ & $\begin{array}{l}\text { Stomach disease, cosmetics } \\
\text { to treat black spots and } \\
\text { freckles }\end{array}$ & Flowers & ++ \\
\hline & Tussilago farfara $\mathrm{L}$. & Coltsfoot & Мати й мачуха & $f l, l$ & Tincture & $\begin{array}{l}\text { Asthma, tuberculosis, stomach } \\
\text { ailments, cosmetics for hair }\end{array}$ & - & + \\
\hline
\end{tabular}


Table 5 Use of medicinal herbs in the Roztochya (Ukraine) (Continued)

\begin{tabular}{|c|c|c|c|c|c|c|c|c|}
\hline CRUCIFERAE & $\begin{array}{l}\text { Capsella bursa-pastoris } \\
\text { (L.) Medik. }\end{array}$ & Shepard's purse & Грицики звичайні & ap & Infusion & Blood coagulation & - & + \\
\hline \multirow[t]{5}{*}{ ERICACEAE } & $\begin{array}{l}\text { Arctostaphylos uva-ursi } \\
\text { (L.) Spreng. }\end{array}$ & Bearberry & Медвежі вушка & I & Infusion & $\begin{array}{l}\text { Kidneys, uterine bleeding, } \\
\text { women's } \\
\text { diseases }\end{array}$ & - & + \\
\hline & Calluna vulgaris (L.) Hull & Heather & Bepec & ap & Honey & Asthma, rheumatism & - & + \\
\hline & Vaccinium myrtillus L. & Bilberry & Чорниця & $b, 1$ & Infusion, food & Vitamins, diabetes, stomach & Fruits & +++ \\
\hline & Vaccinium oxycoccos L. & Cranberry & Клюква & $b$ & Eaten fresh, jams & Vitamins & Fruits & + \\
\hline & $\begin{array}{l}\text { Vaccinium vitis-idaea } \\
\text { (L.) Avror }\end{array}$ & Cowberry & Брусниця & $b, 1$ & Juice, infusion & Vitamins, kidneys problems & Fruits & + \\
\hline GENTIANACEAE & $\begin{array}{l}\text { Centaurium erythraea } \\
\text { Rafn }\end{array}$ & Common centaury & Центорія & $\mathrm{fl}, \mathrm{ap}$ & Infusion & Stomach ailments & - & + \\
\hline GRAMINEAE & Elymus spp. & Couch grass & Пирій & I & Tincture & Urinary tract & - & + \\
\hline GUTTIFERAE & Hypericum perforatum $\mathrm{L}$. & St John's worth & Звіробій & $\mathrm{fl}$, ap & Tincture, infusion & $\begin{array}{l}\text { Stomach ailments (when } \\
\text { stomach hurts), inflammations }\end{array}$ & - & +++ \\
\hline HIPPOCASTANACEAE & Aesculus hippocastanum L. & Chestnut & Каштан кінський & $\mathrm{fl}$ & Tincture & Arthritis & - & + \\
\hline \multirow[t]{6}{*}{ LABIATAE } & Mentha spp. & Mint & М'ята & ap & Tincture, infusion & Toothache, sedative, analgesic & - & ++ \\
\hline & Glechoma hederacea $\mathrm{L}$. & Ground-ivy & Розхідник & । & Infusion & Cold, caught, tuberculosis & - & + \\
\hline & Lamium album $\mathrm{L}$. & White nettle & Духмяна кропива & $\mathrm{fl}$ & tea & $\begin{array}{l}\text { Uterine bleeding, after having } \\
\text { baby }\end{array}$ & - & + \\
\hline & Origanum vulgare $\mathrm{L}$. & Oregano & Материнка & $\mathrm{fl}$, ap & Tincture & $\begin{array}{l}\text { Gynaecological disease } \\
\text { (emmenagogue), breathing } \\
\text { problems }\end{array}$ & - & + \\
\hline & Prunella vulgaris $\mathrm{L}$. & Common self-heal & Суховершки & $\mathrm{fl}$ & Tincture & For throat rinsing & - & + \\
\hline & Thymus spp. & Thyme & $\begin{array}{l}\text { Чебрець, чабрець, } \\
\text { цебрик }\end{array}$ & ap & $\begin{array}{l}\text { Liquids with alcohol, } \\
\text { tincture, infusion }\end{array}$ & $\begin{array}{l}\text { Antiseptic, blood pressure } \\
\text { reduction, anti-hypertension }\end{array}$ & - & ++ \\
\hline LEMNACEAE & Lemna minor $\mathrm{L}$. & Common duckweed & Ряска & ap & Infusion & Psoriasis, asthma & - & + \\
\hline \multirow[t]{2}{*}{ LILIACEAE } & Allium ursinum $L$. & Bear's garlic & Черемша & ap & Tincture, infusion & $\begin{array}{l}\text { Antiseptic, stomach infections, } \\
\text { rheumatism }\end{array}$ & - & + \\
\hline & Convallaria majalis $\mathrm{L}$. & Lily of the valley & Конвалія & $\mathrm{fl}$ & tinctures & Heart disease & - & + \\
\hline LORANTHACEAE & Viscum spp. & Mistletoe & Омела & 1 & Tincture & Blood pressure, immunostimulant & - & + \\
\hline LYCOPODIACEAE & $\begin{array}{l}\text { Huperzia selago (L.) } \\
\text { Bernh. ex Schrank \& Mart., }\end{array}$ & Northern firmoss & Баранець & ap & Tincture & Alcoholism treatment & - & + \\
\hline ONAGRACEAE & Epilobium angustifolium L. & Fireweed & Іван Чай & । & Infusion & Headache, blood coagulation & - & + \\
\hline PAPAVERACEAE & Chelidonium majus L. & Greater celandine & Чистотіл & ap & Tincture & Cancer treatment & - & + \\
\hline
\end{tabular}


Table 5 Use of medicinal herbs in the Roztochya (Ukraine) (Continued)

\begin{tabular}{|c|c|c|c|c|c|c|c|c|}
\hline PINACEAE & Pinus sylvestris L. & Pine & Сосна звичайна, сосна & bd & Tincture with alcohol & $\begin{array}{l}\text { Cough, asthma, cold, } \\
\text { vitamin C }\end{array}$ & - & + \\
\hline PLANTAGINACEAE & Plantago major $\mathrm{L}$. & Greater plantain & Подорожник & ap & Tincture & $\begin{array}{l}\text { Stomach ailments, breathing } \\
\text { problems (tuberculosis) }\end{array}$ & - & + \\
\hline POLYGONACEAE & Polygonum aviculare L. & Common knotgrass & Спориш звичайний & ap & Infusion & Urinary tract, gastritis, kidneys & - & + \\
\hline PRIMULACEAE & Primula veris $\mathrm{L}$. & Common cowslip & Первоцвіт & ap & Tincture, infusion & Cough & - & + \\
\hline \multirow[t]{13}{*}{ ROSACEAE } & Crataegus spp. & Hawthorn & Глід & $f, f l$ & Tincture & $\begin{array}{l}\text { Heart, i.e., hypertension or } \\
\text { regulation of blood pressure, } \\
\text { sedative }\end{array}$ & Fruits & + \\
\hline & Fragaria vesca L. & Strawberry & Суниці лісові, суниці & $b, l, f$ & Tincture & $\begin{array}{l}\text { Flu and cough remedy, } \\
\text { vitamins }\end{array}$ & Fruits & +++ \\
\hline & Potentilla alba L. & White cinquefoil & Перстач білий & r, ap & Tincture & Treatment of thyroid & - & + \\
\hline & $\begin{array}{l}\text { Potentilla erecta } \\
\text { (L.) Hampe }\end{array}$ & Common tormentil & Перстач прямостоячий & r & Tincture & Gastritis, peptic ulcer & - & + \\
\hline & Potentilla reptans $L$. & Creeping cinquefoil & Перстач повзучий & ap & Tincture & Blood coagulation & - & + \\
\hline & Prunus padus L. & Hackberry & $\begin{array}{l}\text { Черемха звичайна, } \\
\text { черемха }\end{array}$ & $\mathrm{f}, \mathrm{ba}$ & Infusion, tincture & Astringent & Fruits & + \\
\hline & Prunus spinosa $\mathrm{L}$. & blackthorn & Терен & $f$ & $\begin{array}{l}\text { Liquids with alcohol, } \\
\text { tincture }\end{array}$ & Stomach disease, depurative & Fruits & + \\
\hline & Pyrus communis $\mathrm{L}$. & European pear & Лісова грушка & $\mathrm{br}, \mathrm{fl}$ & Infusion & Treatment of joints & Fruits & + \\
\hline & Rubus ideaus L. & Raspberry & Малина & l,b & Fresh, frozen, tea, tincture & $\begin{array}{l}\text { Flu and cough remedy, } \\
\text { high } \\
\text { temperature }\end{array}$ & Fruits & +++ \\
\hline & Rosa spp. & Dog rose & Шипшина & $\mathrm{fl}$ & & $\begin{array}{l}\text { Vitamins, immunity improving } \\
\text { (immunostimulant) tea }\end{array}$ & Fruits & ++ \\
\hline & Rubus spp. & Wild blackberries & Види ожин, ожина & $b$ & Juice, infusion (f) & $\begin{array}{l}\text { Vitamins, food, wine } \\
\text { producing }\end{array}$ & Fruits & +++ \\
\hline & Sanguisorba officinalis $\mathrm{L}$. & Great burnet & Родовик & $r$ & Tincture & $\begin{array}{l}\text { Blood cleaning blood } \\
\text { coagulation }\end{array}$ & - & + \\
\hline & Sorbus aucuparia L. & Rowan & $\begin{array}{l}\text { Горобина звичайна, } \\
\text { горобина }\end{array}$ & $b$ & Juice, tincture & Blood pressure normalization & Fruits & + \\
\hline THYMELAEACEAE & Daphne mezereum L. & Mezereon & $\begin{array}{l}\text { Вовчі ягоди, вовче } \\
\text { лико }\end{array}$ & ba & Poisonous, use externally & Arthritis, cold & - & + \\
\hline TILIACEAE & Tilia spp. & Linden & Липа & $\mathrm{fl}$ & Infusion & Vitamins, flu, antipyretic & - & ++ \\
\hline
\end{tabular}


Table 5 Use of medicinal herbs in the Roztochya (Ukraine) (Continued)

\begin{tabular}{|c|c|c|c|c|c|c|c|c|}
\hline UMBELLIFERAE & $\begin{array}{l}\text { Levisticum officinale } \\
\text { W.D.J.Koch }\end{array}$ & Lovage & Любисток & ap & Tincture & $\begin{array}{l}\text { Cosmetics (herb for bath), } \\
\text { skin, stomach, antiseptic,Liver, } \\
\text { kidneys Cosmetics }\end{array}$ & - & + \\
\hline URTICACEAE & Urtica dioica L. & Stinging nettle & $\begin{array}{l}\text { Кропива дводомна, } \\
\text { кропива жалка, кропива }\end{array}$ & I & Infusion, tincture & $\begin{array}{l}\text { Vitamins, cosmetic, blood } \\
\text { coagulation, mouth cleaning } \\
\text { (mouth wash/ oral hygiene) }\end{array}$ & Leaves & + \\
\hline
\end{tabular}


Table 6 Use of medicinal herbs in the Kortkeros (Russia)

\begin{tabular}{|c|c|c|c|c|c|c|c|c|}
\hline Family & Species & English name & Local names & $\begin{array}{l}\text { Part } \\
\text { used }\end{array}$ & Mode of preparation & Medicinal use & Food & $\begin{array}{l}\text { Level of } \\
\text { use }\end{array}$ \\
\hline BETULACEAE & Betula spp. & Birch & Берёза & $\mathrm{bd}, \mathrm{sa}$ & $\begin{array}{l}\text { Liquids with alcohol, } \\
\text { tincture }\end{array}$ & $\begin{array}{l}\text { Panacea ('100 diseases') } \\
\text { e.g., small scratches, flu }\end{array}$ & - & ++ \\
\hline CAPRIFOLIACEAE & Viburnum opulus L. & Guelder rose & Калина обыкновенная калина & $b$ & Infusion, tincture & Vitamins, cough & Fruits & + \\
\hline \multirow[t]{7}{*}{ COMPOSITAE } & Achillea millefollium L. & Yarrow & Тысячелистник обыкновенный & $\mathrm{l}, \mathrm{fl}$ & Infusion & Blood coagulation & - & + \\
\hline & Arctium lappa L. & Greater burdock & Лопух & r & Decoction & $\begin{array}{l}\text { For strengthening hair } \\
\text { (conditioner) }\end{array}$ & - & + \\
\hline & Artemisia absinthium L. & Wormwood & Полынь & $\mathrm{l}, \mathrm{fl}$ & Tea & Stomach ailments & - & + \\
\hline & $\begin{array}{l}\text { Chamomilla recutita } \\
\text { (L.) Rauschert }\end{array}$ & Chamomile & $\begin{array}{l}\text { Ромашка аптечная, } \\
\text { ромашка }\end{array}$ & $\mathrm{l}, \mathrm{fl}$ & Infusion & Good for health & - & + \\
\hline & $\begin{array}{l}\text { Taraxacum officinale } \\
\text { Weber ex Wiggers }\end{array}$ & Dandelion & $\begin{array}{l}\text { Одуванчик лекарственный, } \\
\text { одуванчик }\end{array}$ & $\mathrm{l}, \mathrm{fl}$ & Infusion, tincture & Vitamins, headache & - & + \\
\hline & Tussilago farfara $\mathrm{L}$. & Coltsfoot & Мать-и-мачеха & $f l, l$ & Infusion & Cough & - & + \\
\hline & Tanacetum vulgare $\mathrm{L}$. & Tansy & Пижмо & $|, f|$ & Tincture & Stomach ailments, diarrhea & - & + \\
\hline CRASSULACEAE & Rhodiola rosea $\mathrm{L}$. & Golden root & Золотий корінь & r & Tincture (d) & General 'for good health' & - & + \\
\hline CUPRESSACEAE & Juniperus communis L. & Common juniper & Можжевельник & r & Tincture & For good health & - & + \\
\hline EQUISETACEAE & Equisetum spp. & Horstail & Хвощ & I & Infusion & Kidneys and urinary tract & - & + \\
\hline \multirow[t]{4}{*}{ ERICACEAE } & Ledum palustre L. & Wild rosemary & Трава богульник & ap & $\begin{array}{l}\text { Tincture (must be } \\
\text { stored for } 3 \text { years, } \\
\text { otherwise poisonous) }\end{array}$ & Asthma & - & + \\
\hline & Vaccinium myrtillus L. & Bilberry & Черника & $b$ & Tincture & $\begin{array}{l}\text { Vitamins, eyes disease, } \\
\text { problems with stomach }\end{array}$ & Fruits & +++ \\
\hline & Vaccinium oxycoccos L. & Cranberry & Клюква & $b$ & $\begin{array}{l}\text { Eaten fresh, jams, } \\
\text { frozen }\end{array}$ & $\begin{array}{l}\text { High blood pressure, } \\
\text { vitamins }\end{array}$ & Fruits & +++ \\
\hline & $\begin{array}{l}\text { Vaccinium vitis-idaea } \\
\text { (L.) Avror }\end{array}$ & Cowberry & Брусника & $b, 1$ & Infusion & $\begin{array}{l}\text { Vitamins, flu, kidneys, } \\
\text { diuretics }\end{array}$ & Fruits & +++ \\
\hline GRAMINEAE & Elymus spp. & Couch grass & Пырей & r & Tincture & $\begin{array}{l}\text { Kidneys, breathing problems, } \\
\text { reproductive system }\end{array}$ & - & + \\
\hline GROSSULARIACEAE & Ribes spp. & $\begin{array}{l}\text { Blackcurrant, } \\
\text { redcurrant }\end{array}$ & Смородина & $b, 1$ & F: infusion, juice & Rich in vitamins & - & ++ \\
\hline GUTTIFERAE & Hypericum perforatum $\mathrm{L}$. & St John's worth & Зверобой & I (d) & Tea, tinctures & Problems with stomach & - & + \\
\hline \multirow[t]{2}{*}{ LABIATAE } & Mentha spp. & Mint & Мята & $\mathrm{l}, \mathrm{fl}$ & Tincture, infusion & Nervousness, stomach & Leaves & + \\
\hline & Thymus spp. & Thyme & Тимьян & $l, f l$ & Infusion (f, d) & $\begin{array}{l}\text { For strong health, } \\
\text { immunostimulant }\end{array}$ & - & + \\
\hline LILIACEAE & $\begin{array}{l}\text { Polygonatum odoratum (Mill.) } \\
\text { Druce }\end{array}$ & $\begin{array}{l}\text { Angular Solomon's } \\
\text { seal }\end{array}$ & Купина & $|, f|$ & Tincture & Rheumatism, diabetes & - & + \\
\hline MENYANTHACEAE & Menyanthes trifoliata L. & Bog-bean & Трилисник & । & Tea & Stomach, gastritis & - & + \\
\hline ONAGRACEAE & Epilobium angustifolium L. & Fireweed & Иван-чай & I & Infusion & Cancer treatment & - & + \\
\hline
\end{tabular}


Table 6 Use of medicinal herbs in the Kortkeros (Russia) (Continued)

\begin{tabular}{|c|c|c|c|c|c|c|c|c|}
\hline ORCHIDACEAE & Cypripedium calceolus L. & $\begin{array}{l}\text { Lady's slipper } \\
\text { orchids }\end{array}$ & Венерин башмачок & r & Tea & Headache & - & + \\
\hline PAEONIACEAE & Paeonia anomala L. & Paeonia & Сибірський піон & r & Tincture & For good health & - & + \\
\hline PINACEAE & Abies alba Mill. & Silver fir & Пихта & $b d$ & $\begin{array}{l}\text { Brooms for sauna, } \\
\text { steam bath }\end{array}$ & Immunostimulant & - & + \\
\hline PLANTAGINACEAE & Plantago major L. & Greater plantain & Подорожник & I & Tincture & Small scratches & - & + \\
\hline \multirow[t]{7}{*}{ ROSACEAE } & Comarum palustre L. & Purple marshlocks & Сабельник & $|, f|$ & Tincture & Arthritis, rheumatism, cancer & - & + \\
\hline & Filipendula vulgaris Moench & Dropwort & Таволга & $f l, l$ & Infusion, tincture & $\begin{array}{l}\text { Antiseptic, arthitis, } \\
\text { heart, skin disease }\end{array}$ & - & + \\
\hline & Prunus padus L. & Hackberry & $\begin{array}{l}\text { Черёмуха обыкновенная, } \\
\text { черёмуха }\end{array}$ & $\mathrm{fr}$ & $\begin{array}{l}\text { Dried fruits, infusion } \\
\text { from dried fruits }\end{array}$ & Pain in stomach, diarrhoea & Fruits & ++ \\
\hline & Rubus ideaus L. & Raspberry & Малина & $b r, f r, l$ & Tincture & $\begin{array}{l}\text { Vitamins, flu, high } \\
\text { temperature }\end{array}$ & Fruits & ++ \\
\hline & Rosa spp. & Dog rose & Шиповник & $\mathrm{fr}$ & Infusion & $\begin{array}{l}\text { Flu, vitamins, rich in } \\
\text { vitamin C, for immunity }\end{array}$ & Fruits & +++ \\
\hline & Rubus chamaemorus L. & Cloudberry & Морошка & b, fl se & Infusion & Vitamins, flu, cough & Fruits & +++ \\
\hline & Sorbus aucuparia L. & Rowan & Рябина обыкновенная, рябина & $\mathrm{fr}$ & Juice, dried fruits & $\begin{array}{l}\text { High blood pressure, } \\
\text { stomach problems }\end{array}$ & Fruits & +++ \\
\hline SALICACEAE & Salix spp. & Willow & Ива & ba & Infusion & $\begin{array}{l}\text { Natural aspirin, rheumatism, } \\
\text { ostheochondrosis }\end{array}$ & - & + \\
\hline SCROPHULARIACEAE & Euphrasia officinalis L. & Eyebright & Очанка & $\mathrm{r}$ & Infusion (d) & For eyes disease & - & + \\
\hline
\end{tabular}

$a p$ aerial parts (herb), $b$ berries, $b a$ bark, $b d$ buds, br branches, $f$ flowers, fr fruit / leaves, $r$ root, sa sap, se sepals, $f$ fresh, $d$ dried

- No use, + used by up to 10 respondents, ++ 10-20 respondents, +++ more than 20 respondents. Listed illnesses refer to emic categories
- 
like diabetes and hypertension ('high blood pressure') (Table 5). The most common ailments cured with herbal remedies in the Russian study area were rheumatism and arthritis, upper respiratory tract infections (cough and common cold), kidney and urinary tract problems, high blood pressure, blood coagulation problems and different gastrointestinal problems (stomach ailments, inflammation, gastritis) (Table 6). Remarkably, several respondents in the Russian and Ukrainian study areas claimed that a tincture of Amanita muscaria contains anti-carcinogenic properties.

\section{Wild food species collected for commercial purposes}

Selling wild food was a widespread activity in Roztochya and Kortkeros; in Småland on the other hand, people only harvested wild food species for personal use. Many Swedish respondents stated that the sale of wildly collected fruits and mushrooms was important for rural livelihoods in the Småland region about 6070 years ago.

The majority of respondents in the Ukrainian and Russian study areas mentioned that the collection of edible NWFPs for sale has become more intensive compared to about 20-25 years ago, prior to the collapse of the Soviet Union. During the Soviet period, people had a job at the collective farms, forestry or in the industry and there was neither time nor a need to collect NWFPs to get an additional income. However, collective farms and many industries were closed during the 1990s after the collapse of the Soviet Union. At the time of this study, unemployment in both regions was high. The forest provided an interesting opportunity to support local livelihoods. Accordingly, the majority of interviewees in the Ukrainian and Russian study areas collected fruits and mushrooms for selling. In Roztochya people sold NWFPs on local markets to consumers mainly from urban areas. The most frequently collected fruits for sale were: wild strawberries, bilberries, blackberries and raspberries. The most popular mushrooms collected for sale were the penny bun or cep [Boletus edulis Bull.], redcapped scaber stalk [Leccinum aurantiacum (Bull. ex St. Amans)] and honey fungus [Armillaria spp.]. In Roztochya, the distance to markets varied from two to $60 \mathrm{~km}$. In villages located close to the border with Poland, locals often sold wild fruits (mostly bilberries) to Polish companies, who then transported the fruits to Poland for the production of value-added products. According to Stryamets et al. [18], villagers earn about the equivalent of two monthly salaries in rural areas per season from selling wild fruits (approximately 300 EUR). By picking and selling fruits, respondents could easily earn more than the average daily labour payment in rural areas (approximately 10 EUR).
In Kortkeros local people sold harvested NWFPs mainly to companies that freeze fruits and mushrooms for further transport. Additionally, as the nearest town with a market was up to $60-120 \mathrm{~km}$ away, in each village there were places where people sold their NWFPs to each other and to rare tourists. The respondents mainly sold fruits (bilberries, cowberries, cloudberries and cranberries). Locally collected penny bun and chanterelle were mainly sold at collecting stations to company representatives. The amount of fruits that were sold during the season in Kortkeros varied a lot, depending on the annual yield and on a collector's employment status. Respondents pointed out that they generally collected as much as possible. Several respondents stated that they used their vacation time to pick wild fruits and mushrooms for selling. The minimum sold amount of fruits reported was $100 \mathrm{~kg}$ of cowberries. The maximum was $6 \mathrm{t}$ of bilberries and cowberries (sold by one family in one season) and 1,5 t of mushrooms. Some people earned up to 250000 rubles (approx. 5336 EUR) per season (the maximum sum that was mentioned in 2013).

\section{Discussion}

Wild food and medicine for recreation and economic survival

In all three study areas, interviewees highly appreciated wild food. However, our results indicate that the role of NWFPs as a source of wild food and medicine has a different importance for rural residents in the studied cases.

In Sweden, the current use of wild food and medicine mainly serves recreational purposes. However, historically NWFPs - except for mushrooms - were extensively used as wild food and medicine for centuries [71]. The custom of eating mushrooms was imported from France and adopted by the Swedish nobility in the $18^{\text {th }}$ century. Peasants began using mushrooms as a free food resource only after World War I and many species were used also for medicine $[15,19]$. Our Swedish case study reflects a general trend in Western Europe, where there is a growing interest and demand for organic and natural products provided by forests [3, 72]. In Sweden there is a growing market for medicinal herbs in drug stores as there has been a strong upswing in the dietary supplement market. Nevertheless as other studies also indicated rural people do not collect NWFPs for commercial purposes, and most value-added products from NWFPs sold in Sweden, are produced in other countries [73].

In Sweden, the process of urbanization is one of the fastest in Europe [74]. This rapid urbanization is one of the reasons that many people have become disconnected from nature, which has been proven to cause more stress [75, 76]. Diseases caused by stress and physical inactivity are currently one of the main reasons of illness 
in the developed world [76-78]. At the same time, many scientific studies have shown that interactions with nature can provide a positive influence on mental and physical health and self-awareness [77, 79-81]. Moreover, multiple studies have indicated that activities undertaken in forests reduce stress [82-84] and the amount of the stress hormone cortisol [85]. Wild food collection and consumption provide healthier food, physical activity and stress relief. Most respondents in the Swedish study area indeed stated that collecting wild food has a very positive effect on their mental health. It helps them to reduce stress and provides a break from their daily routine, as collecting NWFPs is accompanied by physical activity, fresh air, relaxation and a general enjoyment of nature. Thus, these forest-based activities provide preventive, treatment and therapeutic health benefits [86, 87].

By contrast, the Ukrainian and Russian case studies illustrate how wild food and medicine can be important for personal food security and as an additional - and in many cases, the only - financial income. Due to political, social and economic developmental challenges in countries transitioning from planned to market economies in Eastern Europe, forest functions, other than wood production, have remained or regained great local and regional importance in the study areas. In Ukraine and NW Russia the use of NWFPs as wild food and traditional medicine has been important for centuries, and for the larger part of the $20^{\text {th }}$ century $[35,48,88,89]$. NWFPs were particularly important during famines in the $19^{\text {th }}$ and $20^{\text {th }}$ centuries in Ukraine $[6,35]$ and because of food scarcity during the $20^{\text {th }}$ century in the Republic of Komi [48, 49]. Currently rural residents in both Roztochya and Kortkeros use NWFPs to supplement their diets and household income, notably during certain seasons of the year, and to help meet medical treatment needs. These resources are important for subsistence and as an additional income during hard times of economic transition, especially considering the high level of unemployment in these rural areas. Thus, NWFPs were important to avoid poverty in the Russian and Ukrainian rural study areas, which has also been shown to be the case in other parts of the world [42, 90-93]. By offering supplemental income (both subsistent and economic), collecting wild food and medicine provides a safety net or a risk management activity during economic crises [46, 90, 94, 95]. According to Chukwuone and Okeke [96] NWFPs contribute to food security in two ways (1) through direct consumption of NWFPs for local diets and (2) through trade of NWFPs to generate income. This view was corroborated by interviews in the Russian study area, which indicate that people survived during economic crisis in the 1990s using NWFPs. Currently, rural households' dependence on wild fruits and mushrooms in Russia is much higher than in Sweden and Ukraine, which is also shown by other studies [72, 97].

The use of wild food and medicine may be related to the socio-economic situation in a country. In a country like Sweden with high living standards and a modern and freely available health care system, the collection, processing and consumption of wild medicine among respondents is limited and has a mainly recreational character. On the other hand, in countries of Eastern Europe such as the Ukraine and Russia which are in transition from planned to market economy with political and economic crises, financial insecurity and expensive medical care, the use of both wild food and medicine is still very active $[13,18]$. In fact the local livelihood strategies of rural forest dependent communities are often based on the use of NWFPs.

\section{Traditional knowledge of wild food and medicine: lost in time?}

Despite the importance for recreation or subsistence, in all three study areas traditional knowledge related to NWFPs was reported to be decreasing, due to shifts in lifestyles and interests. Nearly $90 \%$ of the respondents said that their parents had taught them to pick fruits and mushrooms. Most respondents indicated that nowadays the younger generations appear to have lost interest in forest activities such as collecting foods and medicines. In all three case studies mainly middle-aged and elderly respondents were interested in harvesting and collecting NWFPs, especially if the practice was a tradition in their families and they had lived permanently in the countryside. In the Ukrainian and the Russian study areas, knowledge about medicinal plants was transmitted from generation to generation. The use of medicinal herbs was more popular among the elderly, while the younger generation seemed to be losing interest in using these NWFPs in both study areas. In Ukraine there is a high interest in wild medicinal herbs, for example observed in several TV and radio programs that describe herbs and their uses. The respondents also mentioned so-called 'green pharmacies', pharmacies that only sell medicinal herbs (dried herbs, tinctures and mixes) and provide free consultations on how to use traditional medicines.

Our results are consistent with findings on changes in patterns of wild food and medicine use in other parts of the world $[3,42,89,96]$ that are associated with lifestyle changes, urbanization, large-scale farming, and less contact with nature $[17,37]$. These rapid changes are a threat to traditional practices, and stress the need to document the traditional knowledge that still exists in rural areas of Eastern Europe [98, 99].

Our case studies in Ukraine and Russia indicate that traditional knowledge on preserving wild fruits and 
mushrooms for winter times are still actively maintained. Such traditional methods of winter preserves stem from the past when this was a useful tradition to ensure food security in harsh winter times [3, 13, 36, 37, 100], as these techniques of drying, freezing, marinating and making jams all improve shelf-life considerably [101]. Finally, our findings also suggest that traditional knowledge has a tendency to 'survive' much easier where it has an important role in subsistence such as in Ukraine and Russia (self-sufficiency), as opposed to Sweden [102].

\section{Conclusions}

Our results indicate that the collection of wild food and medicine depends to a large extent on the socioeconomic situation of the collector. In economically less developed rural areas like Roztochya and Kortkeros, collecting wild food and medicine continues to be an important part of local livelihoods. In these areas, NWFPs help ensure food security both directly and by providing an additional source of income. In these case studies the low quality and high costs of biomedical health care were brought up as main reasons for an increase in the consumption of medicinal herbs. In an economically more developed region such as Småland, collecting wild food is mainly a recreational activity with important stress-reducing health benefits. The promotion of wild food and medicine can be a 'bridge' between nature and people. The commercial and health benefitting potential of wild food and medicine could be considerably enhanced by drawing upon traditional knowledge and building on the sustainable system of use that local people often have created. Thus, NWFPs and their value-added processing have potential to support different dimensions of rural livelihoods in Europe.

\section{Endnotes}

${ }^{1}$ In Småland participants generally provided the species name, while in Roztochya and Kortkeros respondents just reported the genus but not always the species of the collected mushrooms. Thus, the total number of collected species might be higher. For instance Russula spp. includes more than 17 species growing in the Roztochya area, but was reported as one genus.

\section{Competing interests}

The authors declare that they have no competing interests.

\section{Authors' contributions}

NS is a leading author that has designed the research and conducted the field study, made the data analysis, wrote the paper. ME was involved in the data collection in the Ukrainian study area, worked on the design of this study together with the leading author, was engaged in the data analysis and writing the paper. MC, PA, RA contributed to the development of manuscript on different stages, read and approved the final manuscript. PA raised the funds for the study. All authors read and approved the final manuscript.

\section{Acknowledgements}

We are grateful to all the interviewees who shared their experience, lifestyle and knowledge. The Swedish Institute and FORMAS provided financial support. We thank Mikael Angelstam for translating interviews in Småland and Mike Manton for his comments. We also thank two anonymous reviewers for their valuable comments.

\section{Author details}

${ }^{1}$ Swedish University of Agricultural Sciences, Forest-Landscape-Society Research Group, Faculty of Forest Sciences, School for Forest Management, PO Box 43, Skinnskatteberg SE 73921, Sweden. ${ }^{2}$ Research Institute for Nature and Forest, Kliniekstraat 25, Brussels 1070, Belgium. ${ }^{3}$ Nature reserve "Roztochya", Sitchovuh Strilciv 7, Ivano-Frankove 81070, Ukraine.

Received: 24 October 2014 Accepted: 12 May 2015

Published online: 16 June 2015

\section{References}

1. Smith-Hall C, Larsen HO, Pouliot M. People, plants and health: a conceptual framework for assessing changes in medicinal plant consumption. J Ethnobiol Ethnomed. 2012;8(43):1-11. doi:10.1186/1746-4269-8-43.

2. Price L. Wild food plants in farming environments with special reference to Northeast Thailand, food as functional and medicinal, and the social roles of women. In: Pieroni A, Price LL, editors. Eating and healing: traditional food as medicine. Binghamton: Food Products Press; 2006. p. 65-99.

3. Łuczaj Ł, Pieroni A, Tardío J, Pardo-de-Santayana M, Sõukand R, Svanberg I, et al. Wild food plant use in 21 st century Europe: the disappearance of old traditions and the search for new cuisines involving wild edibles. Acta Soc Bot Pol. 2012;81(4):359-70. doi:10.5586/asbp.2012.031.

4. FAO. Towards a harmonized definition of non-wood forest products. Unasylva. 1999;50(198):63-4.

5. Saastamoinen O, Kangas K, Aho H. The Picking of Wild Berries in Finland in 1997 and 1998. Scand J For Res. 2000;15(6):645-50. doi:10.1080/ 02827580050216897.

6. Ryabchuk V. Nederevni resursy lisy. [Non-wood forest resources]. Svit: Lviv; 1996.

7. Wong J, Thornber K, Baker N. Resource assessment of non-wood forest products. Experience and biometric principles. Non-food forest products. Food and agriculture organization of the United Nations: Rome; 2001.

8. Saastamoinen O. Forest policies, access rights and non-wood forest products in northern Europe. Unasylva. 1999;50(198):20-6.

9. Quave CL, Pardo-de-Santayana M, Pieroni A. Medical Ethnobotany in Europe: From Field Ethnography to a More Culturally Sensitive Evidence-Based CAM? eCAM. 2012;2012:156846. doi:10.1155/2012/156846.

10. Schulp CJE, Thuiller W, Verburg PH. Wild food in Europe: A synthesis of knowledge and data of terrestrial wild food as an ecosystem service. Ecol Econ. 2014;105:292-305. doi:10.1016/j.ecolecon.2014.06.018.

11. Mabey R. Food for free. London: Harper Collins; 2001.

12. Reynolds Whyte S, van der Geest S, Hardon A. Social Lives of Medicines. Cambridge: Cambridge University Press; 2002.

13. Łuczaj $Ł$, Köhler P, Pirożnikow E, Graniszewska M, Pieroni A, Gervasi T. Wild edible plants of Belarus: from Rostafiński's questionnaire of 1883 to the present. J Ethnobio Ethnomed. 2013;9(21):1-17. doi:10.1186/1746-4269-9-21.

14. Svanberg I. Folklig botanik. [Folk botany]. Dialogos: Stockholm; 2011.

15. Svanberg I, Sõukand R, Łuczaj Ł, Kalle R, Zyryanova O, Dénes A, et al. Uses of tree saps in northern and eastern parts of Europe. Acta Soc Bot Pol. 2012;81(4):343-57. doi:10.5586/asbp.2012.036.

16. Sõukand R, Quave CL, Pieroni A, Pardo-de-Santayana M, Tardio J, Kalle R, et al. Plants used for making recreational tea in Europe: a review based on specific research sites. J Ethnobiol Ethnomed. 2013;9(1):58. doi:10.1186/1746-4269-9-58.

17. Kalle R, Sõukand R. Historical ethnobotanical review of wild edible plants of Estonia (1770s-1960s). Acta Soc Bot Pol. 2012;81(4):271-81. doi:10.5586/ asbp.2012.033.

18. Stryamets N, Elbakidze M, Angelstam P. Role of non-wood forest products for local livelihoods in countries with transition and market economies: case studies in Ukraine and Sweden. Scand J For Res. 2012;27(1):74-87. doi:10.1080/02827581.2011.629622.

19. Kardell L. Forest berries and mushrooms: an endangered resource? Ambio. 1980;9(5):241-7 
20. Janse $G$, Ottitsch A. Factors influencing the role of Non-Wood Forest Products and Services. Forest Policy and Economics. 2005;7(3):309-19. doi:10.1016/ s1389-9341(03)00068-6.

21. Stryamets N, Elbakidze M, Angelstam P, Axelsson R, editors. Role of non-wood forest products for sustainable development of rural communities in countries with a transition: Ukraine as a case study. IUFRO Landscape Ecology Working Group International Conference. Forest Landscapes and Global Change-New Frontiers in Management, Conservation and Restoration. Bragança. Portugal: Instituto Politécnico de Bragança; 2010.

22. Ghirardini MP, Carli M, del Vecchio N, Rovati A, Cova O, Valigi F, et al. The importance of a taste A comparative study on wild food plant consumption in twenty-one local communities in Italy. J Ethnobiol Ethnomed. 2007;3:22. doi:10.1186/1746-4269-3-22.

23. DuBois TA, Lang JF. Johan Turi's animal, mineral, vegetable cures and healing practices: an in-depth analysis of Sami (Saami) folk healing one hundred years ago. J Ethnobiol Ethnomed. 2013;9:57. doi:10.1186/1746-4269-9-57.

24. Vitalini S, Tome F, Fico G. Traditional uses of medicinal plants in Valvestino (Italy). J Ethnopharmacol. 2009;121(1):106-16. doi:10.1016/j.jep.2008.10.005.

25. González-Tejero MR, Casares-Porcel M, Sanchez-Rojas CP, Ramiro-Gutiérrez JM, Molero-Mesa J, Pieroni A, et al. Medicinal plants in the Mediterranean area: synthesis of the results of the project Rubia. J Ethnopharmacol. 2008;116(2):341-57. doi:10.1016/j.jep.2007.11.045.

26. Molina M, Reyes-Garcla V, Pardo-de-Santayana M. Local Knowledge and Management of the Royal Fern (Osmunda regalis L.) in Northern Spain: Implications for Biodiversity Conservation. American Fern Journal. 2009;99(1):45-55. doi:10.1640/0002-8444-99.1.45.

27. Rigat M, Bonet MA, Garcia S, Garnatje T, Valles J. Studies on pharmaceutical ethnobotany in the high river Ter valley (Pyrenees, Catalonia, Iberian Peninsula). J Ethnopharmacol. 2007;113(2):267-77. doi:10.1016/ j.jep.2007.06.004.

28. Pieroni A, Nebel S, Quave C, Münz H, Heinrich M. Ethnopharmacology of liakra: traditional weedy vegetables of the Arbëreshë of the Vulture area in southern Italy. J Ethnopharmacol. 2002;81:165-85.

29. Ryabchuk V, Zayachuk V, Gorbenko N. O P. Likarsky roslyny lisiv Zahidnogo region Ykrainy [Medical plants in forests in Western Ukraine]. Visnyk NLTY. 2006:32:34-6.

30. Pieroni A, Quave C. Functional foods of food medicines? On the consumption of wild plants among Albanians and Southern Italians in Lucania. In: Pieroni A, Price LL, editors. Eating and healing: traditional foods as medicine Binghamton. NY: Haworth Press; 2006. p. 101-29.

31. Jarić $S$, Popović $Z$, Macukanović-Joćić M, Djurdjević L, Mijatović M, Karadzić $B$, et al. An ethnobotanical study on the usage of wild medicinal herbs from Kopaonik Mountain (Central Serbia). J Ethnopharmacol. 2007;111(1):160-75. doi:10.1016/j.jep.2006.11.007.

32. Menković N, Šavikin K, Tasić S, Zdunić G, Stešević D, Milosavljević S, et al. Ethnobotanical study on traditional uses of wild medicinal plants in Prokletije Mountains (Montenegro). J Ethnopharmacol. 2011;133(1):97-107. doi:10.1016/j.jep.2010.09.008.

33. Leporatti ML, Ghedira K. Comparative analysis of medicinal plants used in traditional medicine in Italy and Tunisia. J Ethnobiol Ethnomed. 2009;5:31. doi:10.1186/1746-4269-5-31.

34. Leporatti ML, Ivancheva S. Preliminary comparative analysis of medicinal plants used in the traditional medicine of Bulgaria and Italy. J Ethnopharmacol. 2003;87(2-3):123-42. doi:10.1016/s0378-8741(03)00047-3.

35. Komendar V. Likarski roslyny Karpat. [Medical herbs of the Carpathians] Karpaty: Uzhgorod; 1971

36. Łuczaj Ł. Dzikie rośliny jadalne używane w okresach niedoboru żywności we wschodniej części Karpat (powiaty Krosno, Sanok, Lesko, Nadwórna, Kosów i Kołomyja) według ankiety szkolnej z 1934 roku. [Wild edible plants used in times of food shortage in the eastern part of the Carpathians (districts Krosno, Sanok, Lesko, Nadvirna, Blackbirds and Kołomyja) according to the school survey in 1934]. In: Łuczaj Ł, editor. Dzikie rośliny jadalne - zapomniany potencjał przyrody; 13 września Przemyśl-Bolestraszyce Bolestraszyce: Arboretum i Zakład Fizjografii w Bolestraszycach; 2007. p. 161-81.

37. Łuczaj Ł, Szymanski WM. Wild vascular plants gathered for consumption in the Polish countryside: a review. J Ethnobiol Ethnomed. 2007;3:17. doi:10.1186/1746-4269-3-17.

38. Yelin Y, Zerov M, Lushpa V, Shabarova S. Daru lisiv. [Gifts of the forests] 4th ed. Urozaj: Kyiv; 1987.

39. Ziervogel JM. Pharmacopoeia Holmeniensis Galeno - Chymica 1686 av. The Stockholm Pharmacopoeia: Stockholm; 1979.
40. Backman E. Om den äldsta svenska farmakopén, dess medicinskt-historiska bakgrund och dess ställning till folkmedicinen. [lf the oldest Swedish Pharmacopoeia, its medical-historical background and its position on folk medicine]. Uppsala Läkareförenings Förhandlingar; 1924.

41. Grape A. Linné, Abraham Bäck och Pharmacopoea Svecica av år 1775. [Linnaeus, Abraham Back and Pharmacopoea Svecica of 1775]. Svenska Linnésällskapets Årsskrift Uppsala: 1946.

42. Dénes A, Papp N, Babai D, Czúcz B, Molnár Z. Wild plants used for food by Hungarian ethnic groups living in the Carpathian Basin. Acta Soc Bot Pol. 2012;81(4):381-96. doi:10.5586/asbp.2012.040.

43. Łuczaj Ł. Ethnobotanical review of wild edible plants of Slovakia. Acta Soc Bot Pol. 2012;81(4):245-55. doi:10.5586/asbp.2012.030.

44. Boa E. Wild Edible Fungi: A Global Overview of their Use and Importance to People. Non-wood forest products. Food and Agriculture Organization: Rome; 2004

45. Tibuhwa DD. Wild mushroom-an underutilized healthy food resource and income generator: experience from Tanzania rural areas. J Ethnobiol Ethnomed. 2013;9:49. doi:10.1186/1746-4269-9-49.

46. Elbakidze M, Angelstam P. Implementing sustainable forest management in Ukraine's Carpathian Mountains: The role of traditional village systems. For Ecol Manag. 2007;249(1-2):28-38. doi:10.1016/j.foreco.2007.04.003.

47. World Bank. http://data.worldbank.org/country/. 2014

48. Kotelina T. Gryby tajgy i tyndru. [Fungi of taiga and tundra]. Syktyvkar Komi kn. Izd-vo; 1990.

49. Pearson M, Ojanen $\mathrm{P}$, Havimo $M$, Kuuluvainen $T$, Vasander $H$. On the European Edge- Journey through Komi Nature and Culture. Publications from the Departament of forest ecology. Helsinki: University of Helsinki; 2007.

50. Encyklopediya lekarstvennyh rastenyi. Ukrainska encyklopediya [Encyclopedia of medical plants] Kiev: Golovna redakciya YRE; 1992.

51. Lagerås $P$. The ecology of expansion and abandonment. Medieval and post-medieval land-use and settlement dynamics in a landscape perspective. Riksantikvarieämbetet: Stockholm; 2007.

52. Lagerås P. Vegetation and land-use in the Småland Uplands, southern Sweden, during the last 6000 years Lund: Lund University. 1996.

53. Swedish Forest Agency. Skogsstatistik årsbok. [Forest statistic yearbook]. Jönköping: Skogsstyrelsen 2010

54. Sweden S. Statistical Yearbook of Sweden. Statistics Sweden: Stockholm; 2011

55. Anell A, Glenngård A, Merkur S. Sweden: Health system review. Health Systems in Transition. 2012;14(5):1-159.

56. Stryamets G, Ferenc N. Osoblyvosti rostu introdukovanyh derevnyh vudiv u zapovydnuh umovah [Features of growth of introduced tree species in a protected environment]. Visnyk Lysotehnychnogo universytetu. 1999:9(9):244-50.

57. Parchuk G, Bovt Y, Stryamets G, Stryamets N, Gorban I, Kosyk L. Terytorial'na struktura ta zonuvannya biosfernogo rezervatu "Roztochya". The territorial structure and zoning of the biosphere reserve "Roztochya"]. Naukoviy Vesnik National Forestry University of. Ukraine. 2010;20(16):80-6.

58. Elbakidze M, Angelstam PER, Sandström C, Stryamets N, Crow S, Axelsson R, et al. Biosphere Reserves for conservation and development in Ukraine? Legal recognition and establishment of the Roztochya initiative. Environ Conserv. 2013:40(02):157-66. doi:10.1017/s0376892912000434.

59. Anon. Statystuka Yavorivskogo rayina. [Statistic of the Yavoriv district] Yavoriv: 2008.

60. Anon. Statistika Lvivskoij oblasti [Statistic of the Lviv region]. Lviv: Golovne ypravlinnya statystyku u Lvivskii oblasti; 2014.

61. Shestyukova T. Lesnoe hozjaistvo respubliki Komi [Forestry in Komi Republic]. Komistat: Syktyvkar; 2012.

62. Anon. Statistika Kortkeroskogo rayona. [Statistik of Kortkeros region] Kortkeros: Kortkeroskaya rajonnaya administraciya; 2013.

63. Kvale S. Doing Interviews. Qualitative Research Kit. California: SAGE Publications Ltd, Thousand Oaks; 2007.

64. Kvale S, Brikman S. InterViews: Learning the craft of qualitative research interviewing. 2-nd ed. California: Sage Publications Inc., Thousand Oaks; 2008.

65. Saunders MNK. Choosing research participants. The Practice of Qualitative Organizational Research: Core Methods and Current Challenges. London: Sage; 2012.

66. American anthropological association. Code of ethics. http:// ethics.aaanet.org/category/statement/. 2012. 2013.

67. International Society of Ethnobiology. International Society of Ethnobiology Code of Ethics (with 2008 additions) 2006 
68. Flora Europaea [database on the Internet]. Accessed: 14 July 2014

69. Species 2000 [database on the Internet]. http://www.sp2000.org/. 2014. Accessed: 13 July 2014

70. Red Data Book of Ukraine. Chervona knyga Ukrainy. Roslynnyi svit. [Red Data Book of Ukraine]. Kiev: Globalconsalting; 2009.

71. Yamin-Pasternak S. Ethnomycology: Fungi and Mushrooms in Cultural Entanglements. Ethnobiology. New Jersey: Wiley; 2011. p. 213-30.

72. Turtiainen M, Nuutinen T. Evaluation of information on wild berry and mushroom markets in European countries. Small-scale Forestry. 2011;11(1):131-45. doi:10.1007/s11842-011-9173-z.

73. Messerer M, Johansson SE, Wolk A. Use of dietary supplements and natural remedies increased dramatically during the 1990s. J Intern Med. 2001;250(2):160-6. doi:10.1046/j.1365-2796.2001.00872.x.

74. Eurostat European Commission. Eurostat regional yearbook Brussels2013.

75. Louv R. Last Child in the Woods: Saving Our Children From Nature-Deficit Disorder Updated and Expanded edition ed. Chapel Hill, New York: Algonquin Books; 2008.

76. Selhub EM, Logan AC. Your brain on nature: the science of nature's influence on your health, happiness and vitality. Missisauga: John Wiley \& Sons; 2012.

77. Grahn P, Stigsdotter UK. The relation between perceived sensory dimensions of urban green space and stress restoration. Landsc Urban Plan. 2010;94(3-4):264-75. doi:10.1016/j.landurbplan.2009.10.012.

78. WHO. Depression. Programmes and Projects. Mental Health. World Health Organization. 2008. http://www.who.int/topics/mental_health/en/.

79. Ottoson J. The Importance of Nature in Coping. Alnarp: Swedish University of Agricultural Sciences; 2007

80. Li Q. Forest medicine. New York: Nova; 2012.

81. Sempik J, Hine R, Wilcox D. Green Care: A Conceptual Framework,

82. Morita E, Fukuda S, Nagano J, Hamajima N, Yamamoto H, Iwai Y, et al. Psychological effects of forest environments on healthy adults: Shinrin-yoku (forest-air bathing, walking) as a possible method of stress reduction. Public health. 2007;121(1):54-63. doi:10.1016/j.puhe.2006.05.024.

83. Hine R. Peacock J, J eP. Measuring the Benefits: Green Spaces; 2008.

84. Simoens I, Thoonen M, Meiresonne L, Van Daele T. Ecosysteemdienst groene ruimte voor buitenactiviteiten. [Ecosystem Service of green space for outdoor activities]. Mededelingen van het Instituut voor Natuur- en Bosonderzoek: Brussel; 2014.

85. Custers M, en van den Berg A. Natuur, stress en cortisol: Experimenteel onderzoek naar de invloed van tuinieren en activiteiten in groenkamers op het fysiologisch, affectief en cognitief herstel van stress. [Nature, stress and cortisol: Experimental research on the influence of gardening and activities in green rooms on the physiological, affective and cognitive recovery from stress]. Wageningen 2007.

86. Park B-J, Furuya K, Kasetani T, Takayama N, Kagawa T, Miyazaki Y. Relationship between psychological responses and physical environments in forest settings. Landsc Urban Plan. 2011;102(1):24-32. doi:10.1016/j.landurbplan.2011.03.005.

87. Bowler DE, Buyung-Ali LM, Knight TM, Pullin AS. A systematic review of evidence for the added benefits to health of exposure to natural environments. BMC Public Health. 2010;10:456. doi:10.1186/14712458-10-456.

88. Szot-Radziszewska E. Folk medicine in Ukraine at the end of the 19th and the beginning of the 20th centuries. Analecta - Studia i Materialy z Dziejów Nauki. 1-2(31-32. 2007;16:69-107.

89. de Beer J, Zakharenkov A. Tigers, mushrooms and bonanzas in the Russian Far East: the Udege's campaign for economic survival and conservation. In: Wolvekamp P, editor. Forests for the future: local strategies for forest protection, economic welfare and social justice. London: Zed Books; 1999. p. 244-50.

90. Albers HJ, Robinson EJZ. A review of the spatial economics of non-timber forest product extraction: Implications for policy. Ecol Econ. 2013;92:87-95. doi:10.1016/j.ecolecon.2012.01.021.

91. Arnold M, Ruiz-Perez M. Analysis. Can non-timber forest products match tropical forest conservation and development objectives? Ecol Econ. 2001;39:437-47.

92. Gubbi S, MacMillan DC. Can non-timber forest products solve livelihood problems? A case study from Periyar Tiger Reserve, India. Oryx. 2008;42(02):222-8.

93. Ndoye O, Tieguhong JC. Forest resources and rural livelihoods: the conflict between timber and non-timber forest products in the Congo Basin, Scandinavian Journal of Forest Research, vol Supplement 004. 2004.
94. Jakobsen J. The role of NTFPs in a shifting cultivation system in transition: A village case study from the uplands of North Central Vietnam. Geografisk Tidsskrift-Danish Journal of Geography. 2006;106(2):103-14. doi:10.1080/ 00167223.2006 .10649560$.

95. Pirożnikow E. Rola pożywienia zbieranego z natury w życiu Polaków deportowanych do ZSRR w okresie drugiej wojny światowej. [The role of foraging in the life of Poles deported to the USSR during World War II]. Ethnobiologia Polska. 2014:4:135-72.

96. Chukwuone NA, Okeke CA. Can non-wood forest products be used in promoting household food security?: Evidence from savannah and rain forest regions of Southern Nigeria. Forest Policy and Economics. 2012;25:1-9. doi:10.1016/j.forpol.2012.09.001.

97. Richards R, Saastamoinen O. NTFP policy, access to markets and labour issues in Finland: impacts of regionalization and globalization of the wild berry industry. In: Laird SA, McLain RJ, Wynberg RP, editors. Wild Product Governance: Finding Policies that Work for Non-timber Forest Products London: Earthscan; 2010. p. 287-308

98. Vandebroek I, Reyes-Garcia V, de Albuquerque UP, Bussmann R, Pieroni A. Local knowledge: who cares? J Ethnobiol Ethnomed. 2011;7:35. doi:10.1186/ 1746-4269-7-35.

99. Pieroni A, Privitera S. Ethnobotany and its links to medical sciences and public health: quo vadis? Z Phytother. 2014;35(02):58-62. doi:10.1055/s0034-1371719.

100. Łuczaj Ł. Changes in the utilization of wild green vegetables in Poland since the 19th century: a comparison of four ethnobotanical surveys. J Ethnopharmacol. 2010;128(2):395-404. doi:10.1016/j.jep.2010.01.038.

101. Quave CL, Pieroni A. Fermented Foods for Food Security and Food Sovereignty in the Balkans: A Case Study of the Gorani People of Northeastern Albania. J Ethnobiol. 2014;34(1):28-43. doi:10.2993/02780771-34.1.28.

102. Benz B, Cevallos J, Santana F, Rosales J, Graf S. Losing knowledge about plant use in the Sierra de Manantlan Biosphere Reserve, Mexico. Econ Bot. 2000;54:183-91.

\section{Submit your next manuscript to BioMed Central and take full advantage of:}

- Convenient online submission

- Thorough peer review

- No space constraints or color figure charges

- Immediate publication on acceptance

- Inclusion in PubMed, CAS, Scopus and Google Scholar

- Research which is freely available for redistribution 